\section{Exposição ambiental a interferentes endócrinos com atividade estrogênica e sua associação com distúrbios puberais em crianças}

\author{
Environmental exposure to endocrine disruptors \\ with estrogenic activity and the association with \\ pubertal disorders in children
}

Crésio Alves 1,2

Lindiana Chagas Flores 1

Taís Souza Cerqueira 1

Maria Betânia P. Toralles 1,2

\footnotetext{
${ }^{1}$ Faculdade de Medicina, Universidade Federal da Bahia, Salvador, Brasil. 2 Hospital Universitário Professor Edgard Santos, Universidade Federal da Bahia, Salvador, Brasil.

Correspondência C. Alves Faculdade de Medicina Universidade Federal da Bahia. Rua Plínio Moscoso 222, apto. 601, Salvador, BA 40157-190, Brasil. cresio.alves@uol.com.br
}

\begin{abstract}
Endocrine disruptors are exogenous substances with adverse health effects in intact organisms or their progeny, secondary to changes in endocrine function. Recent years have witnessed constant reports of environmental factors with hormonelike effects causing pubertal or reproductive abnormalities in animals. The few cases proven to be associated with pubertal disorders in humans have been related to accidental exposure. Nevertheless, pediatricians and parents recommend suspending all possible estrogen-contaminated food, especially meat (poultry, beef) and soy products, when the child presents with a pubertal disorder. These recommendations, if not scientifically sound, may have deleterious consequences by eliminating sources of dietary protein and possibly delaying the investigation of other potential and treatable causes. On the other hand, not investigating potential side effects of these products could have similar harmful effects. The current article describes the main endocrine disruptors associated with pubertal disorders in humans and concludes that except for accidental exposure to high doses, more research is needed on the effects of chronic and low-dose exposures in altering human pubertal development.
\end{abstract}

Endocrine Disruptors; Precocious Puberty; Environmental Pollution
Introdução

Puberdade é o período em que o indivíduo desenvolve características sexuais secundárias e progride, através de uma série de estágios, até adquirir função reprodutiva. O mecanismo primário que desencadeia a puberdade ainda não está totalmente esclarecido, mas parece resultar de interações entre fatores genéticos, hormonais e ambientais ${ }^{1}$. No Brasil, o início da puberdade ocorre aos 9,7 anos em meninas e 10,7 anos em meninos 2 . Entretanto, estudos recentes têm mostrado que a puberdade tem iniciado, em média, 1-2 anos antes do que o previamente estabelecido 3,4 .

Embora a tendência secular para redução na idade de início da puberdade possa ser explicada por melhora das condições de vida, a exposição crônica a químicos ambientais tem sido implicada como um dos fatores responsáveis por essa precocidade 5 . A maioria dos estudos nesta área decorre de alterações reprodutivas observadas em animais de laboratório ou animais selvagens provenientes de ecossistemas contaminados 1,6. Informações consistentes apoiando sua ação em humanos são escassas e quase sempre resultado de exposições acidentais.

Esse trabalho tem por objetivo realizar uma revisão crítica da literatura médica sobre a influência de compostos ambientais com atividade endócrina e sua capacidade de alterar a função hormonal e o desenvolvimento puberal de crianças. 


\section{Exposição ambiental a tóxicos e pediatria}

As crianças apresentam um padrão único de vulnerabilidade a produtos tóxicos. Em relação à sua massa corpórea, elas consomem mais água, alimentos e ar que adultos, o que as leva a ter maior exposição a substâncias tóxicas presentes nestes elementos 7 . O seu comportamento de levar a mão e objetos à boca, e o hábito de brincar diretamente ou próximas ao solo ampliam a exposição 7. Devido à imaturidade enzimática, o metabolismo e excreção de substâncias tóxicas são menos desenvolvidos do que nos adultos 8 . $\mathrm{E}$, por terem, potencialmente, mais anos de vida, apresentam maior probabilidade de virem a apresentar conseqüências tardias de exposições cumulativas e prolongadas.

\section{Interferentes endócrinos}

Interferentes endócrinos são sustâncias químicas exógenas, naturais ou sintéticas, com o potencial de causar efeitos adversos na saúde de um organismo ou sua descendência, como resultado de distúrbios na função hormonal 7 . Muitas destas substâncias, embora banidas, ainda permanecem e permanecerão por muito tempo na natureza devido a sua alta estabilidade e, mesmo em pequenas quantidades, seu efeito poderá ser biomagnificado através da ascensão na cadeia alimentar. Não existe um único mecanismo que explique a ação dos interferentes endócrinos uma vez que eles pertencem a diferentes classes. As manifestações clínicas dependem da substância, dose, duração do contato, via de exposição (e.g., in utero, leite materno, oral, parenteral) e período do desenvolvimento no qual a criança foi exposta (e.g., fetal, neonatal, prépuberal, puberal).

A exposição a interferentes endócrinos em períodos críticos do desenvolvimento do feto ou da criança pode causar telarca, pubarca, menarca, ou puberdade precoce; atraso puberal; ginecomastia e malformações reprodutivas (e.g., hipospádia, criptorquidismo) 8 . A descoberta da associação de exposição materna a diestilbestrol e alterações do sistema reprodutor de seus fetos é um marco na história da medicina. Outras vezes, as conseqüências da exposição podem se manifestar 20-40 anos mais tarde através de alterações como diminuição da qualidade do sêmen e endometriose 8 .

Numerosos compostos químicos de uso doméstico, industrial e agrícola possuem comprovada atividade hormonal. Porém, o grau de exposição e efeitos dos outros milhares de produtos com os quais entramos em contato diariamente ainda são desconhecidos.

\section{Interferentes endócrinos e atividade estrogênica}

Com o surgimento de métodos laboratoriais ultra-sensíveis foi demonstrado que meninas prépúberes tem oito vezes mais estradiol que meninos pré-puberes, e que meninas com telarca precoce possuem níveis superiores aos do grupo controle 9 . Ou seja, o estradiol possui, comprovadamente, efeitos biológicos em crianças prépúberes mesmo quando presente em concentrações muito pequenas e não detectáveis pelos métodos tradicionais ${ }^{9}$. Portanto, fontes exógenas de estrógenos podem levar a alterações puberais e reprodutivas em crianças 10 .

Dentre os vários produtos químicos com atividade estrogênica destacam-se: hormônios presentes em cosméticos, anabolizantes utilizados em rações animais, fitoestrógenos e poluentes orgânicos persistentes (POPs). De modo genérico, POPs são compostos lipofílicos, resistentes à degradação, persistentes no ambiente, com capacidade de bioacumulação em pessoas e animais e com toxicidade relevante à saúde humana e ao ambiente 11. Devido a sua estabilidade acabam se perpetuando na cadeia alimentar sendo transportados a grandes distâncias a partir do seu sítio original, até mesmo para regiões onde nunca foram usados, fazendo com que permaneçamos expostos aos resíduos que contaminam a água e alimentos 11,12. Além do efeito hormonal, suas principais ações deletérias são carcinogênese e distúrbios nos sistemas nervoso e imune 8,13 . Os 12 principais POPs (dirty-dozen), descritos inicialmente na Convenção de Estocolmo são: nove agrotóxicos (aldrin, clordano, DDT, dieldrin, endrin, heptaclor, mirex e toxafeno), dois químicos industriais (bifenilas policlorinadas - PCBs - e hexaclorobenzeno) e subprodutos não intencionais (PCBs, dioxinas e furanos, e hexaloroexano) 8,11.

\section{Interferentes endócrinos com efeito no desenvolvimento puberal}

\section{Agrotóxicos}

- DDT

Vários estudos demonstram que o agrotóxico organoclorado diclorodifeniltricloroetano (DDT) possui atividade estrogênica 14 .

O DDT é metabolizado no organismo humano em diversos compostos, sendo os prin- 
cipais o 1,1,1-tricloro-2(p-clorofenil)-2-(oclorofenil)etano (o,p'-DDT), 1,1-dicloro-2-(pclorofenil)-2-(o-clorofenil)etileno (o,p'-DDE), 1,1,1,-tricloro-2,2-bis(p-clorofenil)etano (p,p'DDT) e 1,1,-dicloro-2,2-bis(p-clorofenil)etileno (p,p'-DDE) 15. Os isômeros o,p'-DDT, o,p'-DDE e p,p'-DDT exibem atividade estrogênica comprovada, porém não são estáveis. O derivado p,p'DDE, apesar de não mostrar atividade estrogênica óbvia, é mais estável e lipossolúvel, o que leva a seu acúmulo no tecido adiposo, leite materno e plasma. Por isso, ele é usado como marcador de exposição ao DDT 16.

Estima-se que 90\% do DDT e de seus produtos metabólicos encontrados no organismo dos seres humanos sejam provenientes da alimentação, principalmente pela contaminação de carne bovina, aves, frutas e leite 15 .

Estudo belga mostrou maior prevalência de puberdade precoce em crianças imigrantes oriundas de países em desenvolvimento relacionando esse distúrbio à exposição crônica ao DDT ou seus subprodutos 16. Existem duas hipóteses para o desencadeamento da puberdade precoce após exposição crônica a organoclorados. A primeira, por via periférica, através da estimulação de tecidos sensíveis a estrogênio. A segunda, por via central, por estimulação do hipotálamo que uma vez suspensa levaria à puberdade precoce central 16 .

A Convenção sobre Poluentes Orgânicos Persistentes, realizada em Estocolmo, Suécia (2001) e o governo brasileiro restringem a produção e o uso do DDT a programas de combate a mosquitos vetores de malária e outras doenças transmitidas por artrópodes 11,15. Apesar destas ações, devido a sua persistência ambiental, o DDT continua e continuará por muitos anos a contaminar seres humanos.

\section{- Dieldrin, aldrin, toxafeno}

O dieldrin, o aldrin e toxafeno são agrotóxicos organoclorados com ação estrogênica. Estudo utilizando células mamárias MCF7 concluiu que eles possuíam atividades estrogênicas similares à do DDT, e que seus efeitos também eram cumulativos 17 . Outro trabalho confirmou que o toxafeno funciona como agonista de células miometriais de ratos in vitro 18 . Wade et al. 19 definiram o dieldrin como um organoclorado de ação estrogênica fraca. No entanto, quando misturado ao toxafeno, consegue induzir respostas estrogênicas em menores concentrações que quando usado isoladamente 17. Grande parte do aldrin se transforma em dieldrin pelo processo de decomposição natural 20 .
$\mathrm{O}$ aldrin e o dieldrin acumulam-se no solo por longos períodos, tornando-se disponíveis para o consumo animal por meio de contaminação de pastagens ${ }^{20}$. Também podem se acumular na poeira de tapetes domésticos facilitando a exposição das crianças e são encontrados em sedimentos de rios contaminando bacias hídricas 21. Resíduos de aldrin foram encontrados em lençóis freáticos do rio Atibaia, no Estado de São Paulo 22, e resíduos de dieldrin detectados em amostras de água, peixes e mamíferos da região dos Grandes Lagos na América do Norte 23.

Como estas substâncias são lipofílicas elas têm a tendência de se acumular em tecidos e líquidos com alto teor de gordura 20. Na Espanha, pesquisa em área rural, detectou concentrações excessivas de aldrin, dieldrin e toxafeno no leite materno 24

Embora banidos na década de 90 do século passado, 39 países ainda reportam produção destes pesticidas 20 . No Brasil, a comercialização do aldrin e eldrin foi interrompida em 1985, sendo seu uso permitido somente em campanhas de saúde pública no combate a vetores 25 .

\section{Subprodutos industriais}

\section{- Fitalatos}

Fitalatos são substâncias usadas para dar flexibilidade e durabilidade ao policloreto de vinila (PVC). Eles são encontrados em uma grande variedade de produtos como material médicohospitalar, roupas, cosméticos, detergentes, solventes, óleos lubrificantes e brinquedos 26 . Dois fitalatos, o dietilexil (DEHP) e diisononil (DINP), têm recebido atenção. O DEHP, usado na fabricação de equipamentos médicos, mostrou ser mais tóxico que o DINP, usado para fabricação de brinquedos infantis 17

As principais vias de exposição são contaminação alimentar das embalagens plásticas, aspiração de partículas de aerossol e ingestão de fitalatos por sucção de brinquedos contendo este material ${ }^{17}$. A exposição relacionada a equipamentos médicos se torna importante pela presença de DEHP em materiais como bolsa de armazenar sangue, equipos de infusão parenteral e aparelhos de oxigenação extracorpórea 27.

Alguns componentes do fitalato podem interferir no desenvolvimento mamário por simular a ação do estradiol 26,27. Estudo realizado em neonatos que necessitaram de oxigenação extracorpórea (ECMO) comprovou que os níveis séricos de DEHP nestas crianças foram significativamente maiores que em crianças controle 28. A concentração foi diretamente proporcional à área de superfície do equipamento, 
demonstrando que quanto maior a exposição maior a mensuração sérica. No entanto, nenhum efeito tóxico do DEHP foi demonstrado nas crianças expostas 27. Para avaliar os efeitos em longo prazo após uma exposição aguda, Rais-Bahrami et al. 27 estudaram adolescentes submetidos à oxigenação extracorpórea quando neonatos. $\mathrm{O}$ artigo concluiu que um contato isolado com o DEHP na infância não modifica o desenvolvimento puberal 27.

Embora a exposição aguda não tenha causado problemas, a exposição crônica mostrou ter grande impacto sobre meninas. Em Porto Rico, análise do soro de 41 meninas com telarca precoce e 35 meninas controle identificou níveis elevados de fitalatos em $68 \%$ das meninas com telarca precoce e em apenas $14 \%$ do grupo controle 29 . No Brasil, o uso de fitalatos é permitido na produção de embalagens que possuam contato com alimentos 30 .

\section{- Dioxinas}

Dioxinas são substâncias aromáticas essencialmente produzidas como subproduto de processos industriais, devido à combustão incompleta de compostos orgânicos. Incineradores que processam lixo municipal, resíduo industrial ou material hospitalar; termoelétrica a carvão, fábricas de papel e celulose, fumaça de veículos automotores, cigarro, fornos de produção de cimento e fundição de chumbo são fontes de produção deste composto ${ }^{31}$. Processos naturais como erupções vulcânicas e queimadas florestais são outras fontes desses produtos 32 . Vários estudos têm demonstrado que a dioxina altera o desenvolvimento reprodutivo de animais de laboratório ${ }^{32}$. O homem está exposto à contaminação, de modo direto, por emissões na atmosfera e, modo indireto, por contaminação do solo, alimentos, águas e outros. Em 2000, a União Européia publicou relatório estabelecendo limites para a emissão de dioxinas nos processos de combustão e co-combustão ${ }^{32}$. O Brasil, como signatário da Convenção de Estocolmo, assumiu o compromisso de reduzir gradativamente a emissão destes poluentes na natureza 33 .

\section{- Compostos polibrominados}

Compostos polibrominados (CPB) são usados como retardadores de chama na fabricação de uma variedade de equipamentos tais como aparelhos elétricos, tintas, produtos têxteis e aeronaves 34 . Existem dois tipos de retardadores de chamas, os aditivos, representados pelos difenil-ésteres polibromados (PBDE) e hexabromociclododecano
(HBCD) e os reativos, como o tetrabromobisfenol-A (TBBA). Existem relatos da presença destas substâncias em tecidos humanos, em especial no leite materno de mulheres expostas 35 . A exposição acidental a BPB causou menarca e pubarca precoce em meninas expostas in utero e através do leite materno 36 . Outro estudo mostrou atraso na menarca de meninas expostas a estes compostos 37 .

\section{- Bifenilas policloradas}

Bifenilas policloradas (BPC) são compostos organoclorados que apresentam alta estabilidade térmica e elevada constante dielétrica. Estas propriedades permitiram seu uso na produção de isolantes termoelétricos como: capacitores e transformadores elétricos, bombas de vácuo, turbinas, fluidos hidráulicos, resinas plastificantes, adesivos, aditivo anti-chama, óleos lubrificantes, pesticidas e papel carbono 38,39. As BPC são altamente resistentes à degradação. Elas se acumulam no tecido gorduroso, fazendo com que a exposição ocorra através do consumo de peixe ou outros predadores no topo da cadeia alimentar 5 . Exposição a BPC foi associada a atraso puberal e diminuição do volume testicular em meninos e retardo no surgimento das mamas em meninas 1,40. A utilização desses produtos foi suspensa no final da década de 70 do século passado ${ }^{13}$. No Brasil, as BPC foram comercializadas com o nome de Ascarel a partir de importação dos Estados Unidos e Alemanha, não havendo registros de produção nacional 38 .

\section{- Bisfenol-A}

O bisfenol-A é um composto utilizado na fabricação de plásticos policarbonatos e epóxi de resina, presentes na resina do forro de latas de alimento e bebida, e na composição de material odontológico selante 40 . Ele já foi encontrado no soro de mulheres grávidas, liquido ascítico e amostras de urina 41. Isto mostra que a exposição é universal e a partir de múltiplas fontes, inclusive da água usada para beber ou banhar ${ }^{42}$. Sua atividade estrogênica in vitro não é significante. No entanto, sua ação in vivo depende da espécie exposta. Em ratos da raça Fischer, ele promove síntese de DNA do epitélio vaginal, embora o mesmo não ocorra na raça Sprague-Dawley 43. A exposição pré-natal tem sido associada à ocorrência de câncer no trato reprodutivo e anormalidades fetais em seres humanos 43 .

Outro fenol, o alquilfenol, usado como agente emulsificador e dispersor em numerosos produtos industriais e domésticos (e.g., detergentes e pesticidas), é um potencial interferente endócri- 
no com o qual os seres humanos estão em constante contato 41 .

\section{- Ácido bórico}

Ratos machos expostos a doses elevadas de ácido bórico mostraram alterações na quantidade e mobilidade dos espermatozóides, além de necrose de células germinativas 44 . Sheng et al. 45 relataram que o boro estimula a atividade do receptor 17-beta-estradiol em ratos. Apesar disso, a toxicidade em humanos, causada pela exposição durante as atividades normais, é remota 46 .

\section{Produtos de interesse à saúde e interferentes endócrinos}

\section{Medicamentos}

Pílulas anticoncepcionais e cremes estrogênicos podem estar envolvidos com o desenvolvimento puberal precoce geralmente por exposição acidental. Halperin \& Sizonenko 47 relataram desenvolvimento precoce de mamas em crianças expostas a creme estrogênico. Freni-Titulaer et al. 48 não encontraram associação estatisticamente significante entre exposição doméstica a contraceptivos orais ou cremes de pele e vaginais e desenvolvimento de mamas em meninas. Felner \&White ${ }^{49}$ descreveram ginecomastia e elevações dos níveis de séricos de estradiol após exposição a altas concentrações de estrógenos contidos em cremes tópicos. O uso de cremes tópicos contendo estrógeno na genitália de meninas pré-púberes para tratamento de coalescência de ninfas é outra fonte potencial de contaminação.

\section{Alimentos}

\section{- Carnes}

A utilização de anabolizantes nas rações animais tem por objetivo promover maior ganho de peso, maximizar a produção, aumentar a rentabilidade e diminuir os custos na criação de animais destinados ao abate ${ }^{48}$. No Brasil, o uso de anabolizantes nas rações animais é proibido 49 .

Os anabolizantes animais podem ser hormônios naturais, semi-sintéticos e sintéticos. Os hormônios naturais de uso animal comercializados em nosso país incluem o estradiol (Compudose), testosterona (Sinovex H) e progesterona (Sivonex) 50. O grupo dos anabólicos semi-sintéticos é composto por estibenos, dietil-estil-bestol e hexo-estrol. Os principais compostos sintéticos são: zeranol (atividade estrogênica), acetato de melengestrol (atividade gestagênica) e acetato de trembolone (atividade androgênica) 51 . Em alguns países, o gado criado de forma confinada é tratado com estrógenos (17 $\beta$-estradiol, benzoato de estradiol ou zeranol) isoladamente ou associado a progesterona, testosterona, acetato de trembolone e acetato de melengestrol 51.

A prática de tratar animais de corte com esteróides sexuais pode contribuir para um consumo aumentado destes hormônios não apenas diretamente, a partir da ingestão da carne de animais tratados, mas também via um aumento da excreção destes compostos e seus metabólicos para o ambiente onde eles, teoricamente, poderiam contaminar a água potável 51 .

Em relação à saúde humana, o processo de manufatura na produção de derivados de carne não influencia na concentração e estrutura dos esteróides presentes nos tecidos animais nem protege contra a ingestão destes compostos 52 . Produtos anabólicos podem permanecer na sua forma ativa após o abate e exercer efeito endócrino nos indivíduos que consomem estes alimentos 53 .

Partsch \& Sippel 52 relataram que embora a exposição a estrógenos presentes em carne de animais seja possível, isto só causaria puberdade precoce em humanos se a exposição fosse constante, prolongada, e que a carne fosse proveniente de animais tratados com altas doses de estrógeno. No Brasil nenhum anabolizante é utilizado de forma comercial em galináceos 50 . Apesar disso, desde os relatos de epidemias relacionadas possivelmente a contaminação de carne de frango por estrógenos, alguns pediatras ao atenderem crianças com telarca precoce orientam os pais a suspenderem a ingestão de aves, ovos e seus derivados, atribuindo a esses alimentos a causa do problema.

\section{- Fórmulas à base de soja}

Fitoestrógenos são compostos derivados de plantas com estrutura química e atividade hormonal semelhante ao estradiol. Os principais fitoestrógenos são: isoflavona (genisteína, daidzeína), lignanos e coumestanos. Estas substâncias, principalmente as isoflavonas, podem afetar o sistema reprodutivo, glândulas mamárias, hipotálamo e hipófise. A abundância de fitoestrógenos com ação estrogênica é balanceada pela sua baixa afinidade para os receptores de estrógeno 5,54 .

$\mathrm{Na}$ alimentação da criança tornam-se importantes por estarem presentes nas fórmulas à base de leite de soja 10. Strom et al. 55 estudando adultos expostos a leite de soja quando crianças não encontraram efeitos adversos sobre a saúde 
reprodutiva. A Academia Americana de Pediatria 10 não recomenda restrição ao uso dessas fórmulas, afirmando que fitoestrógenos derivados da soja têm baixa afinidade por receptores de estrógeno e baixa potência estrogênica em bioensaios.

\section{- Leite materno}

Substâncias exógenas lipofílicas que se ligam a proteínas podem ser encontradas no leite materno 56. Deste modo, produtos organoclorados depositados no tecido adiposo podem ser incorporados ao leite de mulheres em período de lactação 13. O encontro de resíduos de DDT em amostras de leite materno de mulheres européias, apesar de sua produção ter sido suspensa em 1970, ilustra o potencial de contaminação desta substância de longa persistência ambiental ${ }^{14}$. Além disso, muitas medicações, incluindo contraceptivos, possuem capacidade de influenciar a atividade hormonal do leite materno 57 . Entretanto, estes relatos são esporádicos e a utilização de contraceptivos orais não é uma contraindicação ao aleitamento materno.

\section{Cosméticos}

Prematuridade sexual tem sido relacionada com exposição acidental ou intencional a cosméticos (e.g., xampu, condicionador, cremes corporais e para cabelos) contendo estrogênio ou extratos placentários 58,59,60. Edidin \& Levitsky 61 descreveram desenvolvimento de ginecomastia após uso de creme capilar contendo estrógeno. Gottswinter et al. 62 relataram aumento de mamas e impotência após o uso de loções para cabelo. A detecção de aumento dos níveis de estradiol e diminuição de testosterona e gonadotrofinas durante a exposição sugere que esta pode ter sido a causa das anormalidades. Outro estudo mostrou que meninas com maturação sexual precoce usavam algum tipo de cosmético com conteúdo hormonal 58. Tiwary 59,60 relatou desenvolvimento sexual precoce após exposição a produtos de cabelo, e que após a suspensão do uso, todos os pacientes tiveram regressão do quadro. O ácido bórico usado na fabricação de cosméticos é outro produto implicado como responsável por essas alterações 46 .

Nos Estados Unidos, não existe controle sobre os cosméticos que contêm estrogênio, não havendo regras fixas para esses produtos 58 . Além disso, algumas empresas não listam os ingredientes nas embalagens, impossibilitando saber se sua composição inclui ou não hormônios 59 .

\section{Exposições ambientais}

Nos últimos anos, várias exposições acidentais a interferentes endócrinos foram descritas como responsáveis por desenvolvimento puberal precoce em crianças ${ }^{1}$. Os principais acidentes foram em Milão (Itália), Barein, Porto Rico e Jerusalém.

\section{Milão}

Scaglioni et al. 63 observaram desenvolvimento precoce de mamas em crianças numa escola de Milão, Itália. Investigação diagnóstica não evidenciou uso de medicamentos, nem presença de estrógeno na alimentação ou na água da piscina. A telarca e a ginecomastia resolveram num período de oito meses. Fara et al. 64 detectaram discreto aumento nos níveis de 17 - $\beta$-estradiol destas crianças, especulando que o desenvolvimento mamário foi causado, provavelmente, por consumo de aves ou carne bovina contaminadas por estrógeno 63. Ainda em Milão, Nizzoli et al. 65 relataram que epidemias de ginecomastia e telarca precoce em crianças que se alimentavam nas lanchonetes de escolas poderiam ser devidas à ingestão de carne de gado que receberam alimentos contendo substâncias estrogênicas.

\section{Barein}

Em 1981, foram descritos oito meninos que desenvolveram ginecomastia numa pequena vila de Barein após ingestão de leite de vaca contaminado com etinilestradiol. Com a suspensão do consumo, a ginecomastia desapareceu espontaneamente 66 .

\section{Porto Rico}

Em 1982, Comas 67 relatou aumento da incidência de telarca, pubarca e puberdade precoce em crianças de Porto Rico. Embora tenha sido postulada uma provável contaminação alimentar, isto não foi confirmado. No mesmo ano, Saenz-de-Rodríguez \& Toro-Sola 68 descreveram 375 pacientes com telarca precoce nas quais $61 \%$ apresentaram cistos ovarianos sugerindo relação entre desenvolvimento dos cistos e exposição prolongada a estrógenos chamando atenção para o uso de zeranol e dietilestibestrol na produção de carnes. Em 1983, Bongiovanni 69 detectou quantidade aumentada de estrógeno em aves, carnes e leite, em Porto Rico. Como possíveis explicações para tais achados ele suspeitou do uso de estrógenos ou produtos anabolizantes nestes animais, ingestão de plantas contendo estrógeno ou seus precursores, ou contaminação de água potável por substâncias de atividade estrogênica. 
Saenz-de-Rodríguez et al. 70 mostraram que a contaminação hormonal de alimentos em Porto Rico possa ser um dos fatores responsáveis pela epidemia de desenvolvimento sexual precoce. Eles demonstraram a presença de níveis significativamente elevados de estradiol na carne de frango local quando comparada com carne de frango dos Estados Unidos.

Em 1986, Freni-Titulaer et al. 48 estudando meninas com telarca precoce detectaram associação significante com o consumo de cereais e frango. Contudo foi considerada a possibilidade desta associação ser devida a uma recordação seletiva graças a declarações prévias na mídia sugerindo a associação do consumo de frango com o desenvolvimento de telarca. Esses mesmos autores observaram associação positiva de telarca precoce com consumo de fórmulas baseadas em soja e produtos derivados de carne e associação negativa com o consumo de cereais.

No último relato sobre a epidemia de precocidade sexual em Porto Rico, Colón et al. 29 afirmaram que a causa da alta incidência de desenvolvimento sexual precoce e suas conseqüências em longo prazo são desconhecidas, atribuindo uma etiologia multifatorial a este processo de desenvolvimento sexual prematuro.

\section{Jerusalém}

Estudo com crianças apresentando desenvolvimento sexual precoce na área de Jerusalém entre 1974 e 1983, e acompanhadas por 3 a 7 anos, mostrou que a incidência de telarca precoce foi elevada no primeiro ano de vida. Embora um fator casual não tenha sido identificado, não foi descartado um possível efeito do consumo de fitoestrógenos 71 .

\section{Conclusão e perspectivas}

Existe grande preocupação que a exposição a interferentes endócrinos ambientais possa estar contribuindo para o decréscimo na idade do iní- cio da puberdade e para ocorrência de puberdade precoce em seres humanos. Embora a maioria dos estudos tenha sido retrospectiva e realizada em animais ou em humanos expostos acidentalmente a grande quantidade desses produtos, não se pode excluir a interferência da exposição crônica a essas substâncias sobre a saúde puberal e reprodutiva. Como existem centenas de interferentes endócrinos, muitas vezes associados em diferentes quantidades, e como não se conhecem em detalhes os mecanismos que levam ao desenvolvimento puberal, a tarefa de investigá-los torna-se muito difícil. Apesar disso, a busca para possíveis interferentes endócrinos está indicada quando não houver um diagnóstico etiológico para o distúrbio puberal.

A identificação dessas substâncias requer o esforço conjunto de pesquisadores, industriais, legisladores e governo no sentido de desenvolver uma agenda de proteção ao meio ambiente que impeça ou reduza a produção de interferentes endócrinos e conseqüentemente minimize as chances de efeitos adversos sobre a função hormonal dos organismos vivos como resultado de tal exposição.

Estudos epidemiológicos prospectivos, principalmente, em locais onde as exposições a esses agentes fossem maior, e estudos de metabolismo hormonal usando animais de laboratório e linhagens celulares seriam importantes para investigar produtos nos quais se suspeita de interferência endócrina.

A recomendação de suspender o consumo de carne de frango, aves ou seus derivados, não encontra respaldo na literatura e pode prejudicar a nutrição da criança ao eliminar uma das fontes protéicas de sua dieta. A presença de químicos no leite materno não significa necessariamente que o mesmo deva ser suspenso, desde que o encontro desses produtos apenas diz sobre sua capacidade de bioacumulação e não sobre sua toxicidade para o lactente. A troca do leite materno por leite de outras espécies ou fórmulas lácteas com adição de água poderia levar a risco até mesmo maiores sobre a saúde da criança. 


\section{Resumo}

A substância exógena que causa efeitos adversos na saúde de um organismo ou sua descendência, como resultado de distúrbios na função hormonal, é denominada interferente endócrino. Nos últimos anos, produtos ambientais com atividades hormonais têm sido documentados como causadores de anormalidades puberais ou reprodutivas em animais. Os poucos casos comprovados em humanos foram aqueles relacionados a exposições acidentais. Apesar disso, pediatras e pais recomendam a suspensão de todos os alimentos potencialmente contaminados, em especial carne (aves, gado) e derivados da soja quando a criança apresenta alguma alteração puberal. Estas recomendações, se não embasadas cientificamente, podem ter conseqüências deletérias, não apenas pela eliminação de fontes protéicas da dieta, como também por retardar a investigação de causas tratáveis. Por outro lado, a não investigação dos efeitos adversos destes produtos é da mesma forma danosa. Esta revisão descreve os principais interferentes endócrinos responsáveis por alterações puberais em humanos e conclui que, excetuando exposições acidentais a altas quantidades destes produtos, mais estudos são necessários para responsabilizar a ação crônica e em baixas doses destas substâncias na alteração do tempo de desenvolvimento puberal em nossa espécie.

Disruptores Endócrinos; Puberdade Precoce; Poluição Ambiental

\section{Referências}

1. Nebesio TD, Pescovitz OH. Historical perspectives. Endocrine disruptors and the timing of puberty. Endocrinologist 2005; 15:44-8.

2. Colli AS. Maturação sexual - referenciais. In: Setian $\mathrm{N}$, organizador. Endocrinologia pediátrica. Aspectos físicos e metabólicos da criança ao adolescente. $2^{\text {a }}$ Ed. São Paulo: Sarvier; 2002. p. 37-44.

3. Herman-Giddens ME, Slora EJ, Wasserman RC, Bourdony CJ, Bhapkar MV, Koch GC, et al. Secondary sexual characteristics and menses in young girls seen in office practice: a study from the Pediatric Research Office Settings Network. Pediatrics1997; 99:505-12.

4. Herman-Giddens ME, Wang L, Koch G. Secondary sexual characteristics in boys: estimates from the National Health and Nutrition Examination Survey III, 1988-1994. Arch Pediatr Adolesc Med 2001; 155:1022-8.

5. Teilmann G, Jull A, Skakkebaek NE, Toppari J. Putative effects of endocrine disrupters on pubertal development in the human. Best Pract Res Clin Endocrinol Metab 2002; 16:105-21.

\section{Colaboradores}

Todos os autores participaram da pesquisa bibliográfica, concepção, planejamento, execução, análise, interpretação dos dados, redação e revisão crítica do trabalho.

\section{Agradecimentos}

Esse trabalho foi parcialmente financiado pela FAPESB (Fundação de Amparo à Pesquisa do Estado da Bahia).
6. Landrigan PJ, Garg A. Chronic effects of toxic environmental exposures on children's health. J Toxicol Clin Toxicol 2002; 40:449-56.

7. Landrigan P, Garg A, Droller DBJ. Assessing the effects of endocrine disruptors in the National Children's Study. Environ Health Perspect 2003; 11:1678-82.

8. Damstra T. Potential effects of certain persistent organic pollutants and endocrine disrupting chemicals on the health of children. Clin Toxicol 2002; 40:457-65.

9. Klein KO, Mericq V, Brown-Dawson JM, Larmore KA, Cabezas P, Cortinez A. Estrogen levels in girls with premature thelarche compared with normal prepubertal girls as determined by an ultrasensitive recombinant cell bioassay. J Pediatr 1999; 134:190-2.

10. Chen A, Rogan WJ. Isoflavones in soy infant formula: a review of evidence for endocrine and other activity in infants. Annu Rev Nutr 2004; 24:33-54. 
11. Stockholm Convention on Persistent Organic Pollutants. http://www.pops.int/documents/convtext/convtext_en.pdf (acessado em 05/Dez/2006).

12. Grigg J. Environmental toxins: their impact on children's health. Arch Dis Child 2004; 89:244-50.

13. Mello-da-Silva CA, Fruchtengarten L. Riscos químicos ambientais à saúde da criança. J Pediatr (Rio J) 2005; 81 Suppl:S205-11.

14. Snedeker SM. Pesticides and breast cancer risk: a review of DDT, DDE, and dieldrin. Environ Health Perspect 2001; 109:35-47.

15. D’Amato C, Torres JPM, Malm O. DDT (dicloro difenil tricloroetano): toxicidade e contaminação ambiental - uma revisão. Química Nova 2002; 25:995-1002.

16. Krstevska-Konstantinova M, Charlier C, Craen M, Caju DM, Heinrichs C, Beautfort C, et al. Sexual precocity after immigration from developing countries to Belgium: evidence of previous exposure to organochlorine pesticides. Hum Reprod 2001; 16:1020-6.

17. Soto AM, Chung KL, Sonnenschein C. The pesticides endosulfan, toxaphene, and dieldrin have estrogenic effects on human estrogen-sensitive cells. Environ Health Perspect 1994; 102:380-3.

18. Hodges LC, Bergerson JS, Hunter DS, Walker CL. Estrogenic effects of organochlorine pesticides on uterine leiomyoma cells in vitro. Toxicol Sci 2000; 54:355-64.

19. Wade MG, Desaulniers D, Leingartner K, Foster WG. Interactions between endosulfan and dieldrin on estrogen-mediated processes in vitro and in vivo. Reprod Toxicol 1997; 11:791-8.

20. Jorgenson JL. Aldrin and dieldrin: a review of research on their production, environmental deposition and fate, bioaccumulation, toxicology, and epidemiology in the United States. Environ Health Perspect 2001; 109:113-39.

21. Doong RA, Sun YC, Liao PL, Peng CK, Wu SC. Distribution and fate of organochlorine pesticide residues in sediments from the selected rivers in Taiwan. Chemosphere 2002; 48:237-46.

22. Suassuna K. Contaminação em Paulínia por aldrin, dieldrin, endrin e outros compostos tóxicos produzidos e descartados pela Shell do Brasil S.A. http://www.greenpeace.org.br/toxicos/pdf/relatorio_shell_gp1.doc (acessado em 12/Mai/2006).

23. Hickey JP, Batterman SA, Chernyak SM. Trends of chlorinated organic contaminants in Great Lakes trout and walleye from 1970 to 1998. Arch Environ Contam Toxicol 2006; 50:97-110.

24. Campoy C, Jimenez M, Olea-Serrano MF, MorenoFrias M, Canabate F, Olea N, et al. Analysis of organochlorine pesticides in human milk: preliminary results. Early Hum Dev 2001; 65:183-90.

25. Santos JS, Xavier AAO, Ries EF, Costabeber IH, Emanuelli T. Níveis de organoclorados em queijos produzidos no Estado do Rio Grande do Sul. Ciênc Rural 2006; 36:630-5.

26. Shea KM. Pediatric exposure and potential toxicity of phthalate plasticizers. Pediatrics 2003; 111:146774.
27. Rais-Bahrami K, Nunez S, Revenis ME, Luban NLC Short BL. Follow-up study of adolescents exposed to di(2-Ethylhexyl) phthalate (DEHP) as neonates on extracorporeal membrane oxygenation (ECMO) support. Environ Health Perspect 2004; 112:133940.

28. Karle VA, Short BL, Martin GR, Bulas DI, Getson PR, Luban NL, et al. Extracorporeal membrane oxygenation exposes infants to the plasticizer, di(2ethylhexyl)phthalate. Crit Care Med 1997; 25:696703.

29. Colón I, Caro D, Bourdony CJ, Rosario O. Identification of phthalate esters in the serum of young Puerto Rican girls with premature breast development. Environ Health Perspect 2000; 108:895-900.

30. Agência Nacional de Vigilância Sanitária. Lista positiva de aditivos para materiais plásticos. Resolução 95/94. http://elegis.bvs.br/leisref/public/ showAct.php?id=15918\&mode (acessado em 03/ Dez/2005).

31. Assunção JV, Pesquero CR. Dioxinas e furanos: origens e riscos. Rev Saúde Pública 1999; 33:523-30.

32. Cabrita I, Gulyurtlu I, Pinto F, Boavida D, Costa P, Racha L. Formação e destruição das dioxinas em processos de combustão e co-combustão. Revista da Faculdade de Medicina de Lisboa 2003; 8:22535.

33. Ministério do Meio Ambiente. Laboratório analisará poluentes orgânicos. http://www.mma.gov. $\mathrm{br} / \mathrm{ascom} / \mathrm{ultimas} / \mathrm{index} . \mathrm{cfm}$ ?id=1502 (acessado em 19/Mai/2006).

34. Eriksson P, Jakobsson E, Fredriksson A. Brominated flame retardants: a novel class of developmental neurotoxicants in our environment? Environ Health Perspect 2001; 109:903-8.

35. Hooper K, McDonald TA. The PBDEs: an emerging environmental challenge and another reason for breast-milk monitoring programs. Environ Health Perspect 2000; 108:387-92.

36. Blanck HM, Marcus M, Tolbert PE, Rubin C, Henderson AK, Hertzberg VS, et al. Age at menarche and tanner stage in girls exposed in utero and postnatally to polybrominated biphenyl. Epidemiology 2000; 11:641-7.

37. Den Hond E, Schoeters G. Endocrine disrupters and human puberty. Int J Androl 2006; 29:264-71.

38. Penteado JCP, Vaz JM. O legado das bifenilas policloradas (PCBs). Química Nova 2001; 24:390-8.

39. Carpenter DO. Polychlorinated biphenyls (PCBs): routes of exposure and effects on human health. Rev Environ Health 2006; 21:1-23.

40. Lemmen JG, Arends RJ, van der Saag PT, van der Burg B. In vivo imaging of activated estrogen in utero by estrogens and bisphenol A. Environ Health Perspect 2004; 112:1544-9.

41. Calafat AM, Kuklenyik Z, Reidy JA, Caudill SP, Ekong J, Needham LL. Urinary concentrations of bisphenol A and 4-nonylphenol in a human reference population. Environ Health Perspect 2005; 113:391-5.

42. vom Saal FS, Hughes C. An extensive new literature concerning low-dose effects of bisfenol A shows the need for a new risk assessment. Environ Health Perspect 2005; 113:926-33. 
43. Milligan SR, Balasubramanian AV, Kalita JC. Relative potency of xenobiotic estrogens in an acute in vivo mammalian assay. Environ Health Perspect 1998; 106:23-6.

44. Kudo S, Tanase H, Yamasaki M, Nakao M, Miyata Y, Tsuru K, et al. Collaborative work to evaluate toxicity on male reproductive organs by repeated dose studies in rats. A comparative 2- and 4-week repeated oral dose testicular toxicity study of boric acid in rats. J Toxicol Sci 2000; 25:223-32.

45. Sheng MH, Taper LJ, Veit H, Qian H, Ritchey SJ, Lau KH. Dietary boron supplementation enhanced the action of estrogen, but not that of parathyroid hormone, to improve trabecular bone quality in ovariectomized rats. Biol Trace Elem Res 2001; 82:10923.

46. Fail PA, Chapin RE, Price CJ, Heindel JJ. General, reproductive, developmental, and endocrine toxicity of boronated compounds. Reprod Toxicol 1998; 12:1-18.

47. Halperin DS, Sizonenko PC. Prepubertal gynecomastia following topical induction of estrogen containing ointment. Helv Paediatr Acta 1983; 38:361-6.

48. Freni-Titulaer LW, Cordero JF, Haddock L, Lebron G, Martinez R, Mills JL. Premature thelarche in Puerto Rico. A search for environmental factors. Am J Dis Child 1986; 140:1263-7.

49. Felner EI, White PC. Pubertal gynecomastia: indirect exposure to estrogens cream. Pediatrics 2000; 105:55-7.

50. Souza MV, Guimarães PTC, Bressan MC, Costa MCG, Ferreira IC, Faria PB. Anabolizantes: uma discussão sem preconceitos. http://www.editora. ufla.br/Boletim/pdfextensao/bol_02.pdf (acessado em 23/Mai/2006).

51. Andersson AM, Skakkebaek NE. Exposure to exogenous estrogens in food: possible impact on human development and health. Eur J Endocrinol 1999; 140:477-85.

52. Partsch CJ, Sippell WG. Pathogenesis and epidemiology of precocious puberty. Effects of exogenous oestrogens. Hum Reprod Update 2001; 7:292-302.

53. Leffers H, Naesby M, Vendelbo B, Skakkebaek NE, Jorgensen M. Oestrogenic potencies of Zeranol, oestradiol, diethylstilboestrol, Bisphenol-A and genistein: implications for exposure assessment of potential endocrine disrupters. Hum Reprod 2001; 16:1037-45

54. Zung A, Reifen R, Keren Z, Zadik Z. Phytoestrogens: the pediatric perspective. J Pediatr Gastroenterol Nutr 2001; 33:112-8.

55. Strom BL, Schinnar R, Ziegler EE, Barnhart KT, Sommel MD, Macones GA, et al. Exposure to soybased formula in infancy and endocrinological and reproductive outcomes in young adulthood. JAMA 2001; 286:807-14
56. Borgert CJ, Lakind JS, Witorsch RJ. A critical review of methods for comparing estrogenic activity of endogenous and exogenous chemicals in human milk and infant formula. Environ Health Perspect 2003; 111:1020-36

57. Grosvenor CE, Picciano MF, Baumrucker CR. Hormones and growth factors in milk. Endocr Rev 1992; 14:710-28.

58. Zimmerman PA, Francis GL. Hormone-containing cosmetics may cause signs of early sexual development. Mil Med 1995; 160:628-30.

59. Tiwary CM. A survey of use of hormone/placentacontaining hair preparations by parents and/or children attending pediatric clinics. Mil Med 1997; 162:252-6.

60. Tiwary CM. Premature sexual development in children following the use of estrogen- or placenta-containing hair products. Clin Pediatr 1998; 37:733-9.

61. Edidin DV, Levitsky LL. Prepubertal gynecomastia associated with estrogen-containing hair cream. Am J Dis Child 1982; 136:587-8.

62. Gottswinter JM, Korth-Schutz S, Ziegler R. Gynecomastia caused by estrogen containing hair lotion. J Endocrinol Invest 1984; 7:383-6.

63. Scaglioni S, Di Pietro C, Bigatello A, Chiumello G. Breast enlargement at an Italian school. Lancet $1978 ; 1: 551-2$.

64. Fara GM, Del Corvo G, Bernuzzi S, Bigatello A, Pietro C, Scaglioni S, et al. Epidemic of breast enlargement in an Italian school. Lancet 1979; 2:295-

65. Nizzoli G, Del Corno G, Fara GM, Chiumello G. Gynaecomastia and premathure thelarche in a schoolchildren population of northern Italy. Acta Endocrinol Suppl (Copenh) 1986; 279:227-31.

66. Kimball AM, Hamadeh R, Mahmood RA, Khalfan S, Muhsin A, Ghabrial F, et al. Gynaecomastia among children in Bahrain. Lancet 1981; 1:671-2.

67. Comas AP. Precocious sexual development in Puerto Rico. Lancet 1982; 1:1299-300.

68. Saenz-de-Rodríguez CA, Toro-Sola MA. Anabolic steroids in meat and premature thelarche. Lancet 1982; $1: 1300$

69. Bongiovanni AM. An epidemic of premature thelarche in Puerto Rico. J Pediatr 1983; 103:245-6.

70. Saenz-de-Rodríguez CA, Bongiovanni AN, Condede-Borrego L. An epidemic of precocious development in Puerto Rican children. J Pediatr 1985 107:393-6.

71. Boneh A, Landau H, Friendlander N. Age and seasonal factors in the incidence of premature sexual development in girls in Jerusalem area. Clin Invest Med 1989; 12:172-4

Recebido em 06/Jul/2006

Versão final reapresentada em 20/Dez/2006 Aprovado em 16/Jan/2007 